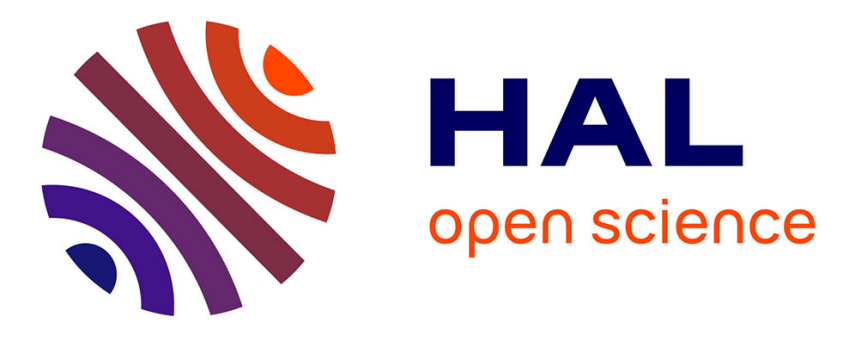

\title{
Longitudinal coherence measurements of a transient collisional x-ray laser
}

Raymond F. Smith, James Dunn, James R. Hunter, J. Nilsen, Sébastien Hubert, Sylvie Jacquemot, Christian Remond, Rémy Marmoret, Marta Fajardo, Philippe Zeitoun, et al.

\section{To cite this version:}

Raymond F. Smith, James Dunn, James R. Hunter, J. Nilsen, Sébastien Hubert, et al.. Longitudinal coherence measurements of a transient collisional x-ray laser. Optics Letters, 2003, 28 (22), pp.22612263. 10.1364/OL.28.002261 . hal-00873446

\section{HAL Id: hal-00873446 \\ https://hal-iogs.archives-ouvertes.fr/hal-00873446}

Submitted on 15 Oct 2013

HAL is a multi-disciplinary open access archive for the deposit and dissemination of scientific research documents, whether they are published or not. The documents may come from teaching and research institutions in France or abroad, or from public or private research centers.
L'archive ouverte pluridisciplinaire HAL, est destinée au dépôt et à la diffusion de documents scientifiques de niveau recherche, publiés ou non, émanant des établissements d'enseignement et de recherche français ou étrangers, des laboratoires publics ou privés. 


\title{
Longitudinal coherence measurements of a transient collisional x-ray laser
}

\author{
Raymond F. Smith, James Dunn, James R. Hunter, and Joseph Nilsen \\ Lawrence Livermore National Laboratory, Livermore, California 94551
}

Sebastien Hubert, Sylvie Jacquemot, Christian Remond, and Remy Marmoret

Commisariat á l'Ènergie Atomique, B.P. 2, 91680 Bruyeres-le-Chatel, France

Marta Fajardo, Philippe Zeitoun, and Laurent Vanbostal

Laboratoire d'Interaction du Rayonnement X avec la Matiére, Université Paris-Sud, Bâtiment 350, 91405 Orsay, France

Ciaran L. S. Lewis

School of Mathematics and Physics, The Queen's University of Belfast, Belfast BT7 1NN, UK

\author{
Marie Francoise Ravet and Franck Delmotte \\ Laboratoire Charles Fabry de l'Institut d'Optique, Université Paris-Sud, Bâtiment 350, 91405 Orsay, France
}

Received June 9, 2003

\begin{abstract}
We present what is to our knowledge the first longitudinal coherence measurement of a transient inversion collisional x-ray laser. We investigated the picosecond output of a Ni-like Pd x-ray laser at $14.68 \mathrm{~nm}$ generated by the COMET laser facility at the Lawrence Livermore National Laboratory. Interference fringes were generated with a Michelson interferometer setup in which a thin multilayer membrane was used as a beam splitter. We determined the longitudinal coherence for the $4 d^{1} S_{0} \rightarrow 4 p^{1} P_{1}$ lasing transition to be $\sim 400 \mu \mathrm{m}$ (1/e half-width) by changing the length of one interferometer arm and measuring the resultant variation in fringe visibility. The inferred gain-narrowed linewidth of $\sim 0.29 \mathrm{pm}$ is a factor of 4 less than previously measured in quasi-steady-state x-ray laser schemes. (C) 2003 Optical Society of America

OCIS codes: $140.7240,030.1640,300.3700,300.6360$.
\end{abstract}

The rapid development of x-ray lasers combined with the availability of optics in the extreme ultraviolet has led to several applications, such as in interferometry ${ }^{1}$ and holography, ${ }^{2}$ that depend on the coherence properties of the source pulse. In amplitude division interferometry, if the phase fronts of the two arms spatially overlap and are copropagated and if the arm lengths are equalized to an accuracy better than the coherence length $L_{c}$ of the source, interference fringes will be generated. The longitudinal coherence of the source is inversely related to the spectral bandwidth $\Delta \lambda$ and is a measure of the temporal separation along a beam in which the different spectral components maintain a phase relationship. This relationship can yield important spectral linewidth and shape information, providing valuable insight into the gain dynamics of the lasing medium. Therefore, accurate measurements of x-ray-laser longitudinal coherence and methods for improving coherence are important for future extremeultraviolet interferometer designs.

Previous measurements of $L_{c}$ were made on the NOVA Ne-like Y x-ray laser at $15.5 \mathrm{~nm} .^{3}$ This quasisteady-state scheme was generated with a single 500 -ps pulse with multikilojoule energy. The longitudinal coherence of this $\mathrm{x}$-ray laser was $\sim 100 \mu \mathrm{m} \mathrm{(1/e}$ half-width), measured with a Mach-Zehnder interferometer with an inferred linewidth of $1.3 \mathrm{pm}$ (FWHM). ${ }^{3}$ In an earlier NOVA experiment, ${ }^{4}$ a 1.1-pm (FWHM) linewidth for the Ne-like Se 20.6-nm x-ray laser, pumped by a 600-ps pulse, was measured directly with a high-resolution spectrometer. In these quasisteady-state schemes the duration of the x-ray laser pulse is shorter than that of the optical drive pulse, where the gain conditions are ultimately extinguished through overheating and overionization of the high-temperature plasma medium.

The transient collisional excitation $\mathrm{x}$-ray $\operatorname{laser}^{5}$ is a higher-efficiency scheme suitable for laser drivers of less than 10-J energy. The strong inversion is pumped by a combination of a long prepulse, of the order of a nanosecond, with a short, picosecond, heating pulse for which the duration of the very high gain $\left(\sim 65 \mathrm{~cm}^{-1}\right)$ is determined by the picosecond time scale over which collisional redistribution of the excited-state populations takes place. The picosecond output makes this scheme well suited for probing fast-evolving events. ${ }^{6}$ In this Letter we report that the transient $\mathrm{x}$-ray laser has an improved longitudinal coherence of $\sim 400 \mu \mathrm{m}$ with an inferred gain-narrowed linewidth of $\sim 0.29 \mathrm{pm}$. This linewidth is approximately four times narrower than that of the NOVA $\mathrm{x}$-ray laser, mainly because of the lower plasma ion temperature in the optimized gain medium.

Saturated lasing at extreme-ultraviolet wavelengths is typically generated through single-pass amplification along an extended laser-produced plasma column. 
The Ni-like Pd 14.68-nm x-ray laser beam was generated by two laser beams at 1054-nm wavelength from the COMET laser facility at the Lawrence Livermore National Laboratory. ${ }^{7}$ Saturated x-ray lasing output of $\sim 10 \mu \mathrm{J}$ was achieved with an optical pumping combination of a 600 -ps-long pulse $\left(2 \mathrm{~J}, 2 \times 10^{11} \mathrm{~W} \mathrm{~cm}^{-2}\right)$ and a $13-p s\left(5 \mathrm{~J}, 3 \times 10^{13} \mathrm{~W} \mathrm{~cm}^{-2}\right)$ main heating pulse. For some shots the duration of the heating pulse was changed to $6 \mathrm{ps}\left(5 \mathrm{~J}, 6 \times 10^{13} \mathrm{~W} \mathrm{~cm}^{-2}\right)$. A 1.6-cm-long line focus that used a traveling-wave geometry irradiated a 1.25-cm-long polished Pd slab target. This irradiation produced strong amplification in one axial direction.

The experimental setup is shown in Fig. 1. The $\mathrm{x}$-ray laser (XRL) output was imaged by a normalincidence Mo-Si multilayer spherical mirror (S1), with $f=11.75 \mathrm{~cm}$, and was routed via a $45^{\circ}$ multilayer mirror to the input path of a Michelson interferometer. The x-ray laser interacts with a thin multilayer foil beam splitter, which consists of an 89-nm-thick $\mathrm{Si}_{3} \mathrm{~N}_{4}$ membrane (area of $0.5 \mathrm{~cm} \times 0.5 \mathrm{~cm}$ ) coated on both sides with 4.5 bilayers of $\mathrm{Mo}-\mathrm{Si}$, giving wavelength reflectivity and transmission of $14 \%$ and $15 \%$, respectively. The two arms, M1 and M2, $34 \mathrm{~cm}$ in length, of the interferometer are defined by normal-incidence $0^{\circ}$ multilayer optics. We generate interference fringes by adjusting the spatial overlap and length of one arm by adjustment of the M2 mirror. The output beam from the interferometer reflects off the M3 mirror onto a spherical multilayer imaging mirror, $\mathrm{S} 2(f=50 \mathrm{~cm})$, and is then relayed via a $45^{\circ}$ multilayer optic to a charge-coupled device (CCD) detector. The x-ray laser's output is effectively relay imaged, via mirrors S1 and S2, to the CCD detector plane with a compound magnification of $\sim 150$. The estimated throughput of the interferometer is approximately 0.007 per arm.

A sample interferogram is shown in Fig. 2 with a lineout that shows the fringe visibility. The image captured by the CCD camera represents a region of $\sim 1700 \mu \mathrm{m} \times 1700 \mu \mathrm{m}$ at the S2 object plane and encompasses most of the exit pattern of the x-ray laser. The observed fringe curvature and variation in the fringe separation are caused by stresses across the beam splitter, which introduce changes to the phase front in the overlapped beams. ${ }^{3}$ Good fringe visibility, $V=\left(I_{\max }-I_{\min }\right) /\left(I_{\max }+I_{\min }\right)$, of as much as $70 \%$ was measured with good continuity across the large-scale intensity variations associated with transverse mode structure in the x-ray laser beam. High-spatial-frequency structure along individual fringes was observed, which may be due to nonuniformities within the interferometer optics. Intensity lineouts for fringe visibility measurements were averaged over a 30-pixel width (Fig. 2), which had the effect of reducing the noiselike contribution from the high-spatial-frequency structure.

We determined the longitudinal coherence of the source by adjusting the differential path length $(\Delta L)$ between the two arms of the interferometer and monitoring the fringe visibility in the interferograms. Measurements were made for both a 6- and a 13-ps laser pulse used to generate the x-ray laser, as shown in Fig. 3. We found that, for increasing values of $\Delta L$, regions in which good fringe quality was observed became more localized, indicating spatial variation of the longitudinal coherence across the x-ray laser beam. Each data point in Fig. 3 signifies the average

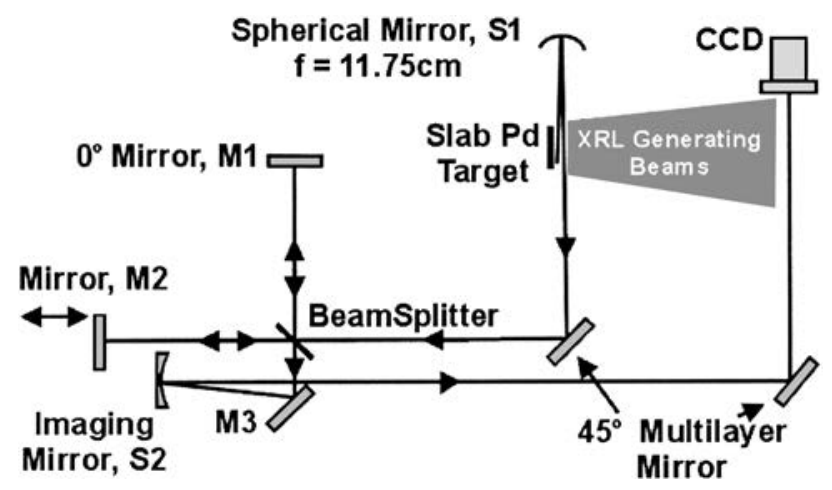

Fig. 1. Schematic of the Michelson interferometer experiment.

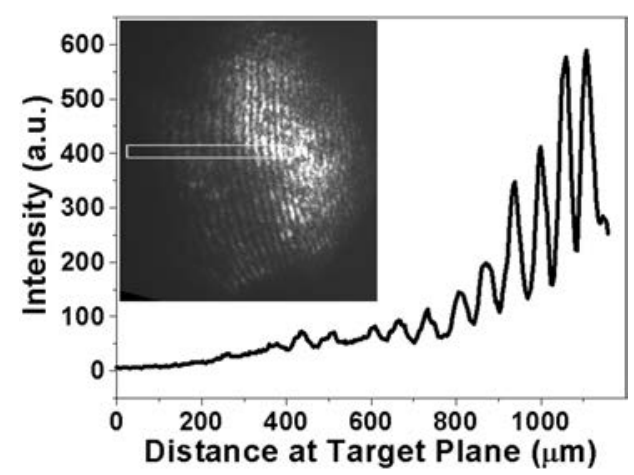

Fig. 2. Interferogram with an intensity lineout taken at $\Delta L=0$ for a 13 -ps heating pulse, showing good fringe visibility over several hundred micrometers.

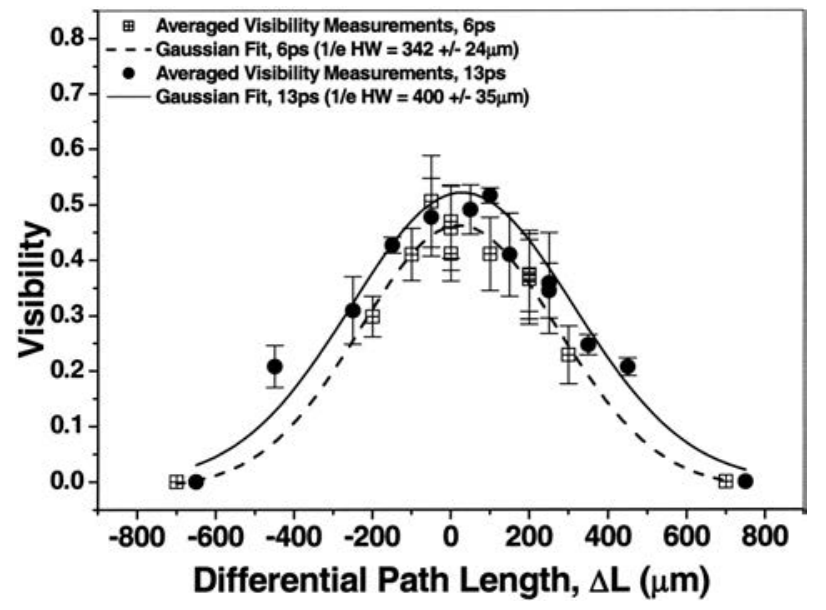

Fig. 3. Variation of fringe visibility with path difference between two arms of the interferometer shown for a 6-ps (open symbols) and a 13-ps (filled symbols) heating pulse. Each data point represents the average visibility for a given shot taken from nine separate measurements across the interferogram. Error bars represent one standard deviation. The experimental points are fitted by Gaussian curves with $1 / e$ half-widths of $400 \mu \mathrm{m} \pm 35 \mu \mathrm{m}$ (solid curve, $13 \mathrm{ps}$ ) and $342 \mu \mathrm{m} \pm 24 \mu \mathrm{m}$ (dashed curve, $6 \mathrm{ps}$ ). 
of the nine highest-visibility measurements across a single interferogram. Ideally at optimum temporal overlap fringe visibility up to $100 \%$ would be observed. In practice this was not achieved because of a small degree of spatial misalignment and slight variations in beam throughput for each arm. Fringe visibility below 0.2 at larger values of $\Delta L$ because the fringe continuity was reduced to a scale less than the integration window. No fringes were observed for $\Delta L$ above $\pm 750 \mu \mathrm{m}$.

From Ref. 8, the measured fringe visibility is equal to the magnitude of the complex degree of coherence within the illumination beam. That degree of coherence is a measure of the degree of correlation between the two combining phase fronts at the output of the interferometer. By use of a Fourier-transform relationship (Wiener-Khintchine theorem) the complex degree of coherence is shown to be related to the power spectral density of the x-ray laser pulse, $I(v)$. As a result of this relationship, linewidth $\Delta v$ (FWHM) and coherence time $\tau_{c}$ are inversely related. We assume that the x-ray laser line has a Gaussian power spectrum such that $I(v) \propto \exp \{-[2 \sqrt{\ln (2)} \times$ $\left.\left.\left(v-v_{0}\right) / \Delta v\right]^{2}\right\}$, where $\Delta v$ is the linewidth of the lasing transition, $v_{0}=c / \lambda$. By increasing the length of arm M2 in increments of distance $L$ we delay the phase front associated with that arm by a time $\tau=2 L / c$. The measured dependence of fringe visibility on $\tau, V(\tau)$, is the envelope of the autocorrelation function of the power spectrum, which varies as $V(\tau)=\exp \left(-\{\pi \Delta v \tau /[2 \sqrt{\ln (2)}]\}^{2}\right)$. The coherence time, $\tau_{c}$, is defined as the value of $\tau$ at which the visibility has decreased to $1 / e$ of the maximum value. Shown in Fig. 3 is a Gaussian fit to the data points with the $1 / e$ half-width yielding a coherence length measurement of $342 \mu \mathrm{m} \pm 24 \mu \mathrm{m}$ for a 6 -ps heating pulse and of $400 \mu \mathrm{m} \pm 35 \mu \mathrm{m}$ for a 13-ps heating pulse. These values are equivalent to spectral linewidths of $\sim 0.34$ and $\sim 0.29 \mathrm{pm}$ (FWHM) for 6 - and 13-ps x-ray laser heating pulses, respectively. The longitudinal coherence measurements deduced by the average visibility plot represents the maximum value from the distribution within the x-ray laser profile. Simulations of the conditions within the $\mathrm{x}$-ray laser medium were carried out with the one-dimensional LASNEX plasma physics $\operatorname{code}^{10}$ for a 13-ps heating pulse. From these calculations we predicted that the ion temperature would remain constant $( \pm 10 \%)$ within the region encompassing the gain profile and a time greater than the gain duration. For the predicted ion temperature of $35 \mathrm{eV}$ the Doppler-broadened line shape is equivalent to $\sim 0.65 \mathrm{pm}$ (FWHM). Gain narrowing of the Doppler-broadened linewidth scales as $\sim(g l)^{-1 / 2}$, where $g$ is the small-signal gain and $l$ is the length of the gain medium. ${ }^{4}$ Previously measured gain-length products of 18 (Ref. 7) would narrow the predicted
Doppler-broadened line shape to $\sim 0.15 \mathrm{pm}$. Other line mechanisms that affect the line shape ${ }^{4}$ would be expected to bring this value into closer agreement with the experimental measurements. The measured linewidth is a factor of 4 less than the $1.3 \mathrm{pm}$ previously measured for the Ni-like Y quasi-steady-state $\mathrm{x}$-ray laser scheme. ${ }^{3}$ In that study the predicted ion temperature of $600 \mathrm{eV}$ was substantially higher than for the plasma conditions reported here, which affects the laser's linewidth and coherence length.

In conclusion, longitudinal coherence measurements have been made of the Ni-like Pd transient x-ray laser that was recently used in the laboratory to interferometrically probe laser-produced plasmas. The good longitudinal coherence achieved, shown for two different driving pulses, is promising for future applications such as in holography.

The support of Al Osterheld and Andy Hazi is greatly appreciated. This research was performed under the auspices of the U.S. Department of Energy by the University of California Lawrence Livermore National Laboratory under contract W-7405-Eng-48. The optics used in the interferometer were funded through the CEA, France. R. F. Smith's e-mail address is smith248@llnl.gov.

\section{References}

1. L. B. Da Silva, T. W. Barbee, Jr., R. Cauble, P. Celliers, D. Ciarlo, S. Libby, R. A. London, D. Matthews, S. Mrowka, J. C. Moreno, D. Ress, J. E. Trebes, A. S. Wan, and F. Weber, Phys. Rev. Lett. 74, 3991 (1995).

2. J. E. Trebes, S. B. Brown, E. M. Campbell, D. L. Matthews, D. G. Nilson, G. F. Stone, and D. A. Whelan, Science 238, 517 (1987).

3. P. Celliers, F. Weber, L. B. DaSilva, T. W. Barbee, Jr., R. Cauble, A. S. Wan, and J. C. Moreno, Opt. Lett. 20, 1907 (1995).

4. J. A. Koch, B. J. MacGowan, L. B. DaSilva, D. L. Mattews, J. H. Underwood, P. J. Batson, and S. Mrowka, Phys. Rev. Lett. 68, 3291 (1992).

5. P. V. Nickles, V. N. Shlyaptsev, M. Kalachnikov, M. Schnurer, I. Will, and W. Sandner, Phys. Rev. Lett. 78, 2748 (1997)

6. R. F. Smith, J. Dunn, J. Nilsen, V. N. Shlyaptsev, S. Moon, J. Filevich, J. J. Rocca, M. C. Marconi, J. R. Hunter, and T. W. Barbee, Jr., Phys. Rev. Lett. 89, 065004 (2002).

7. J. Dunn, Y. Li, A. L. Osterheld, J. Nilsen, J. R. Hunter, and V. N. Shlyaptsev, Phys. Rev. Lett. 84, 4834 (2000).

8. P. W. Milonni and J. H. Eberly, Lasers (Wiley, New York, 1988), pp. 559-562.

9. M. Born and E. Wolf, Principles of Optics, 7th ed. (Cambridge U. Press, Cambridge, 1999), p. 567.

10. J. Nilsen, J. Dunn, R. F. Smith, and T. W. Barbee, Jr., J. Opt. Soc. Am. B 20, 191 (2003). 